\title{
PROPAGATING COUPLED ALFVÉN AND KINK OSCILLATIONS IN AN ARBITRARY INHOMOGENEOUS CORONA
}

\author{
D. J. Pascoe, A. N. Wright, and I. De Moortel \\ School of Mathematics and Statistics, University of St Andrews, St Andrews, KY16 9SS, UK; dpascoe@mcs.st-andrews.ac.uk \\ Received 2010 October 27; accepted 2011 February 16; published 2011 March 24
}

\begin{abstract}
Observations have revealed ubiquitous transverse velocity perturbation waves propagating in the solar corona. We perform three-dimensional numerical simulations of footpoint-driven transverse waves propagating in a low $\beta$ plasma. We consider the cases of distorted cylindrical flux tubes and a randomly generated inhomogeneous medium. When density structuring is present, mode coupling in inhomogeneous regions leads to the coupling of the kink mode to the Alfvén mode. The decay of the propagating kink wave is observed as energy is transferred to the local Alfvén mode. In all cases considered, modest changes in density were capable of efficiently converting energy from the driving footpoint motion to localized Alfvén modes. We have demonstrated that mode coupling efficiently couples propagating kink perturbations to Alfvén modes in an arbitrary inhomogeneous medium. This has the consequence that transverse footpoint motions at the base of the corona will deposit energy to Alfvén modes in the corona.
\end{abstract}

Key words: magnetohydrodynamics (MHD) - Sun: atmosphere - Sun: corona - Sun: magnetic topology - Sun: oscillations - waves

Online-only material: color figures

\section{INTRODUCTION}

Magnetohydrodynamic (MHD) waves in the solar corona attract attention as a possible mechanism for coronal heating in some part of the solar atmosphere (see, e.g., Hollweg 1990; Poedts 2002; Walsh \& Ireland 2003 and references therein), as well as solar wind acceleration, and as a seismological tool for remote diagnostic of coronal plasma parameters (De Moortel 2005; Nakariakov \& Verwichte 2005; Andries et al. 2009). Recent observations of the solar corona by Tomczyk et al. (2007) and Tomczyk \& McIntosh (2009) with the ground-based coronagraph CoMP revealed spatially and temporally ubiquitous propagating transverse velocity oscillations. Their time series of Doppler images revealed waves which are undetected by imaging instruments because of their low amplitude which produces neither significant intensity fluctuations nor resolvable loop displacements. The observed dominance of outward-propagating wave power over inward-propagating wave power along closed loops suggests significant attenuation occurs in situ. The broad power spectrum, peaked at periods of about 5 minutes, hints at driving related to solar $p$-modes. Similar transverse oscillations were observed by Hinode/Solar Optical Telescope in chromospheric structures (De Pontieu et al. 2007) and by Hinode/ $\mathrm{X}$-Ray Telescope in X-ray jets (Cirtain et al. 2007).

Due to their ubiquitous nature, there has been, and still is, much interest in these observed oscillations. Although initially interpreted as Alfvén waves, there has been considerable discussion about their interpretation (see, e.g., Erdélyi \& Fedun 2007; Van Doorsselaere et al. 2008; Goossens et al. 2009). Pascoe et al. (2010) show that propagating transverse velocity waves launched from loop footpoints couple efficiently through mode coupling to Alfvén waves when the medium is non-uniform. The coupling happens within a few wavelengths and drains energy from the driven kink mode oscillation. Observationally, this will be seen as a decay of the driven wave fields, i.e., the transverse oscillations, since the Alfvén mode component, which increases in time, is unresolved by modern instruments.
The study by Pascoe et al. (2010), which was the first to suggest mode coupling, was the key to understanding the data: their work found "the most general interpretation of the observed waves [is] as a coupling of the kink and Alfvén modes." They also "demonstrated that this coupling can account for the predominance of outward wave power in longer coronal loops as observed by Tomczyk \& McIntosh (2009).” Pascoe et al. (2010) also showed how the damping length of the kink mode $\left(L_{d}\right)$ could be estimated for a kink wavepacket in terms of its decay time $(\tau)$ and its group velocity $\left(V_{g}\right)$ :

$$
L_{d}=V_{g} \tau
$$

and found (for the dominant period of $300 \mathrm{~s}$ ) a decay length of $750 \mathrm{Mm}$. They noted that "this simple estimate of damping length scales is therefore consistent with observations: loops that are small (compared to the damping length) will have a stronger inward propagating component than loops that are large (compared to the damping length), or flux tubes that are open." In longer loops, the waves launched from the photosphere will simply not survive long enough to set up a standing wave, so will be seen as propagating waves.

Subsequent studies by Verth et al. (2010) and Terradas et al. (2010) have explored the damped kink mode mechanism in more detail. Terradas et al. (2010) considered the dependence of the damping length on frequency, unlike Pascoe et al. (2010) who only considered the dominant frequency in the data. Terradas et al. (2010) deduced the property that the damping length should be inversely proportional to the wave frequency. Verth et al. (2010) confirmed that this feature was present in data and this has provided compelling evidence for the decay of propagating kink waves as being the correct interpretation of the data; not only is the predicted damping length scale similar to that in observations (Pascoe et al. 2010), but the variation of the damping length with frequency is also seen (Verth et al. 2010).

The rapid damping of global kink standing modes was considered by, e.g., Ruderman \& Roberts (2002) and Goossens 
et al. (2002) in the context of flare-excited, transverse coronal loop oscillations. These models require a finite-width region (of width $l$ ) in which the resonant absorption takes place, extracting energy from the global kink mode. The decay time depends on several variables, such as the density ratio between the coronal loop and its environment, the relative thickness of the inhomogeneity layer, and the oscillation period. Goossens et al. (2002) and Arregui et al. (2007b) applied these models to the observed oscillations to derive constraints on some of these input variables. A review of resonant absorption for standing modes in one-dimensional and two-dimensional models can be found in Goossens et al. (2006) and, more recently, Goossens (2008). The coupling of propagating fast (kink and sausage) modes to Alfvén waves in a waveguide has also received considerable attention in the context of Earth's magnetotail (Allan \& Wright 1998, 2000; Wright et al. 1999; Wright \& Allan 2008). Even though Pascoe et al. (2010) study damping of propagating waves through mode conversion (to be able to relate to the observations of Tomczyk et al. 2007), it was found that the results may closely match the results derived for resonant damping of standing modes. Similarly, Hood et al. (2005) showed that normal mode calculations can be a useful indicator of the behavior of propagating wavetrains when the spatial and temporal scales match (see also Terradas et al. 2010).

The persistence of resonant mode coupling for standing modes in models with structuring in the two-dimensional transverse to the background magnetic field was demonstrated by, e.g., Keppens et al. (1994), Arregui et al. (2007a), Terradas et al. (2008), and Russell \& Wright (2010). These authors showed that resonant absorption in such an inhomogeneous model takes place, not only at the edge of the structure but also at all locations where the resonance condition is satisfied (i.e., where the frequency of the global mode matches the local Alfvén frequency).

The predominance of outward power observed by Tomczyk $\&$ McIntosh (2009) suggests strong attenuation of the velocity oscillations in situ. Hence, a model in terms of propagating waves rather than standing (eigen)modes is more appropriate. So, rather than focusing on standing modes, in this study we investigate the effect of arbitrary transverse inhomogeneities on the coupling between propagating kink and Alfvén modes.

The paper is organized as follows: an overview of the behavior of propagating kink wavepackets is given in Section 2. Our numerical model and driver are described in Section 3. In Section 4, we present our results for the cases of distorted (i.e., non-axisymmetric) cylinders and a randomly generated medium. We discuss our results in Section 5.

\section{DECAY OF PROPAGATING KINK WAVEPACKETS}

In order to put our studies in context, we will first present a brief overview of some existing literature on mode coupling.

The process of resonant absorption was first suggested by Ionson (1978) as a mechanism for heating coronal loops. Since then, there have been numerous studies, both analytical (e.g., Hollweg 1987; Davila 1987) and numerical (e.g., Steinolfson \& Davila 1993; Ofman et al. 1994, 1995), on resonant absorption (see e.g., reviews by Goossens et al. 2006; Goossens 2008).

Many studies consider a single parallel wavenumber component $k_{\|}$, or multiples thereof for higher harmonics, and consider a harmonic driver in time. The model can be reduced to a onedimensional problem by introducing an "azimuthal" wavenumber (e.g., Berghmans \& Tirry 1997). This leads to the "resonant absorption" condition for wave coupling described as where the

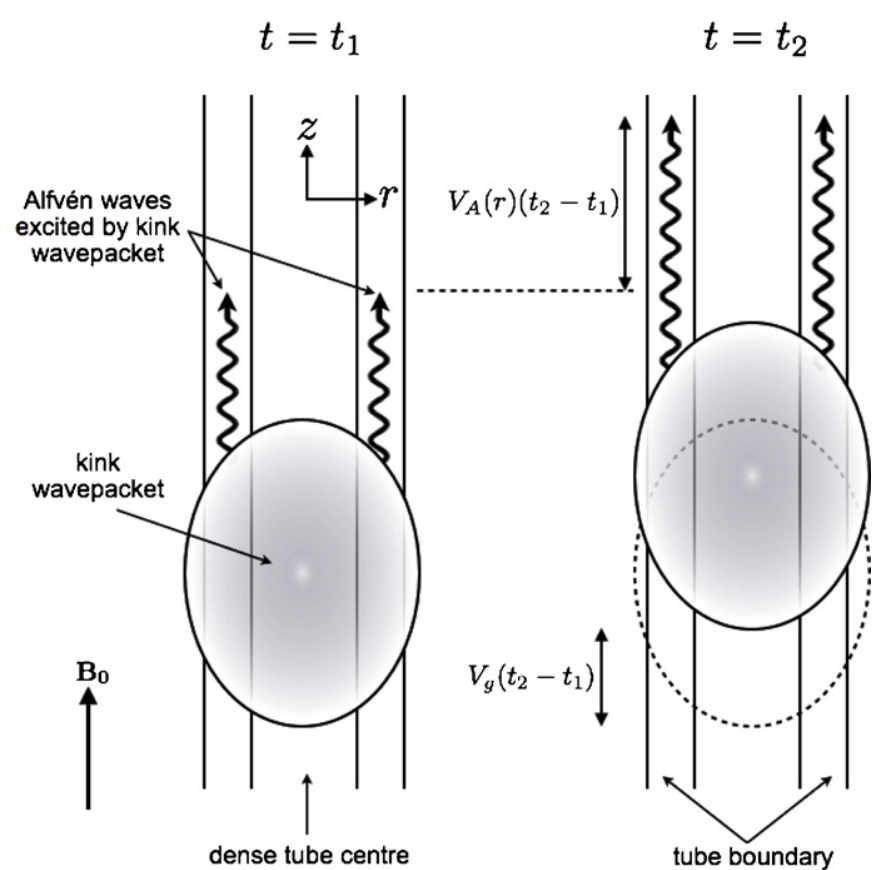

Figure 1. Cartoon of the mode coupling mechanism for propagating kink wavepackets. The wavepacket propagates along the flux tube at a group velocity $V_{g}$ and also has a phase velocity $V_{p}$ associated with it. Mode coupling occurs where $V_{p}$ equals the phase speed of the Alfvén wave $\left(V_{A}(r)\right)$.

driving frequency matches the local Alfvén frequency. If the "azimuthal" modes do not decouple, then a two-dimensional PDE problem results and resonant wave coupling occurs again where the driving frequency matches the local Alfvén frequency (e.g., Tirry et al. 1997; Russell \& Wright 2010). If field-aligned standing modes do not exist, harmonic time solutions (i.e., propagating modes) and/or complex $k_{z}$ solutions may exist. These correspond to damping of compressional modes due to resonant absorption: resonant coupling still occurs where the driving frequency matches the local Alfvén frequency (e.g., Terradas et al. 2010).

Some models relax the assumption of a harmonic time dependence for the driver. If $k_{\|}$is quantized, the perpendicular equilibrium structure supports natural compressional modes with discrete eigenfrequencies. Again, resonant mode coupling occurs where the local Alfvén frequency matches the natural compressional frequencies (e.g., Wright \& Rickard 1995; De Groof \& Goossens 2000, 2002; Terradas et al. 2006).

If we relax the assumption of harmonic studies in time and in $z$, we no longer have the idea of resonant mode coupling, or the notion of driving frequency, local Alfvén frequency, and resonant singularities. The case of wavepacket propagation and dispersion in coronal flux tubes in the absence of mode coupling was first considered by Roberts et al. (1984).

If the tube boundary layer has a finite thickness, we now have a more general time-dependent problem involving wavepacket propagation and decay through mode coupling. There is a rich history of such studies related to mode coupling occurring in the magnetotail (e.g., Allan \& Wright 1998, 2000; Wright et al. 1999; Wright \& Allan 2008). Figure 1 shows the situation where a wavepacket of the kink mode is being guided along the low Alfvén speed central dense flux tube. Such a wavepacket could be excited as a result of footpoint motions disturbing the ends of the flux tube, or as a result of equilibrium reconfigurations due to processes such as reconnection (as in the magnetotail). 
Once the kink wavepacket has been established, it propagates along the flux tube at a group velocity $V_{g}$. The kink wavepacket also has a phase velocity $V_{p}$ associated with it. Allan \& Wright (2000) show that on field lines (in the tube boundary layer) where $V_{A}(r)=V_{p}$, efficient mode coupling between kink and Alfvén waves can occur.

It is important to note that there is no resonant singularity in this process, and there is no harmonic driving frequency (as the kink pulse will provide a broad band of frequencies). The criteria for mode coupling are now based upon matching the phase speed of the kink disturbance $\left(V_{p}\right)$ with the phase speed of the Alfvén wave $\left(V_{A}(r)\right)$.

The Alfvén waves that are excited propagate with their group velocity (also $V_{A}(r)$ ) and run ahead of the kink mode since $V_{A}(r)=V_{p}>V_{g}$ in dense flux tubes. The kink mode is a moving source of Alfvén waves. The picture is similar to someone running along (at $V_{g}$ ) while shining a torch that radiates light ahead of it traveling at a greater speed.

Since the kink mode is losing energy to the Alfvén waves, it will reduce in amplitude as it propagates. The study of the growth of Alfvén energy (which is the focus of magnetotail studies) has been complemented recently by Pascoe et al. (2010) who have studied the damping of the kink wavepacket (in a coronal setting) as it propagates. They also show how a judicious use of the extensive "normal mode" and "resonant" studies can be a useful guide to understanding the behavior of the propagation and damping of kink pulses.

The magnetotail studies show how modes in which the tube axis is not displaced will give rise to similar behavior. These studies also show how a lack of a resonant frequency in wavepacket coupling means there is not a resonant singularity, but rather a broad layer at the tube boundaries in which mode coupling occurs.

Considerable insight into the wave coupling process can be gained from a ray tracing (or WKB) model (Wright et al. 1999) and can be used to interpret the dense coronal flux tube in Figure 1. The magnetoacoustic mode (i.e., kink or sausage) can be described as a superposition of normal modes. A particular mode and radial harmonic (having frequency $\omega$, azimuthal wavenumber $m$, and parallel wavenumber $k_{\|}$) propagates along the ray trajectory as shown in Figure 2. As it encounters the nonuniform Alfvén speed in the tube boundaries it is refracted and turns around (for an axisymmetric tube) at $r_{t}$ where

$$
\frac{\omega^{2}}{V_{A}^{2}\left(r_{t}\right)}=k_{\|}^{2}+\frac{m^{2}}{r_{t}^{2}} .
$$

Beyond $r_{t}$ is the mode coupling location $r_{A}$ defined by

$$
\frac{\omega^{2}}{V_{A}^{2}\left(r_{A}\right)}=k_{\|}^{2} .
$$

Magnetoacoustic perturbations are evanescent beyond $r_{t}$, so the compressional wave energy has to tunnel to the coupling site $\left(r_{A}\right)$. Typically, as $m$ increases the distance between $r_{A}$ and $r_{t}$ increases resulting in an increased tunneling distance and more rapid evanescence. In the case of large $m$, this results in coupling to Alfvén modes becoming negligible. This effect is offset at smaller $m$ by the fact that as $m$ increases, the azimuthal gradients that drive the mode coupling increase. The most efficient coupling typically occurs for $m$ around 3 or 4 (Allan et al. 1986). Note that analytical treatments in which the boundary layer is taken to be asymptotically small (thinboundary or TB approximation) will constrain $r_{t}$ and $r_{A}$ to be

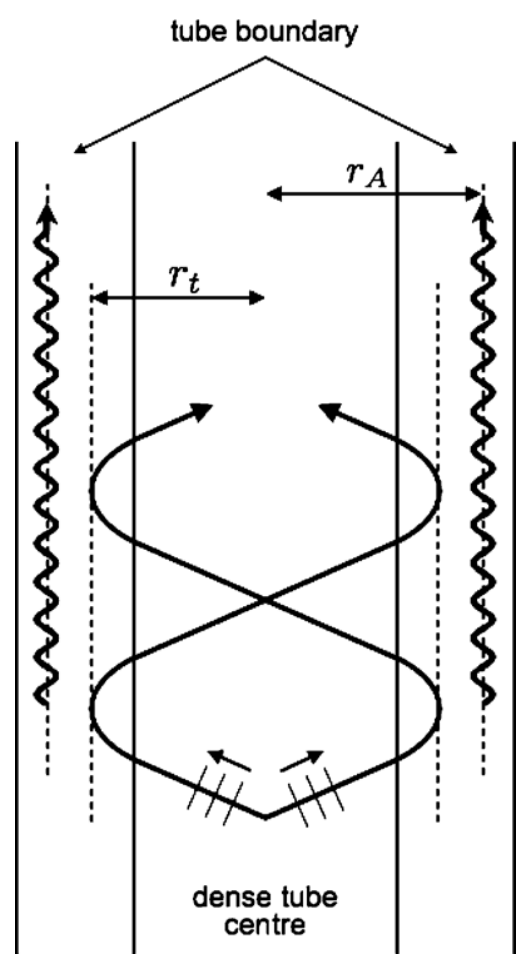

Figure 2. Ray tracing sketch showing the trapping of magnetoacoustic energy in a dense tube with turning point $r_{t}$. Energy can tunnel to $r_{A}$ where it couples to Alfvén waves.

in close proximity. In this case, the coupling efficiency may continue to increase with $m$ (e.g., Goossens et al. 2009). The kink mode has $m=1$.

\section{MODEL}

Considering a global kink standing mode in a zero $\beta$ cylindrical flux tube with an inhomogeneous layer, Ruderman \& Roberts (2002) derived the damping rate by resonant absorption

$$
\frac{\tau}{P}=C \frac{a}{l} \frac{\rho_{0}+\rho_{e}}{\rho_{0}-\rho_{e}},
$$

where $\tau$ is the damping time, $P$ is the period of oscillation, $a$ is the loop radius, $l$ is the inhomogeneous layer thickness, and $\rho_{0}$ and $\rho_{e}$ are the internal and external mass densities, respectively. The constant $C$ depends upon the chosen density profile in the inhomogeneous layer. For a sinusoidal density profile in the inhomogeneous layer $C=2 / \pi$, whereas for a linear density profile $C=(2 / \pi)^{2}$ (see, e.g., Hollweg \& Yang 1988; Goossens et al. 1992; Roberts 2008). Pascoe et al. (2010) found that this relationship also holds in the case of propagating wavetrains with $C=0.9$ for a linear density profile.

\subsection{Driver}

The driving condition is applied to the lower $z$ boundary to simulate excitation by footpoint motions and prescribes the $x$ - and $y$-components of velocity to have a time dependence $f(t)$ :

$$
\mathbf{v}=f(t) \mathbf{u}, \mathbf{u}=\left(u_{x}, u_{y}, 0\right) .
$$

The time dependence of our driver is based on a single period displacement of a flux tube axis. The displacement has the form $\xi \propto \sin (\omega t)$ combined with an envelope of $\sin \left(\frac{\omega}{2} t\right)$ to provide a smooth ramp up and down at the beginning and end of the driving 


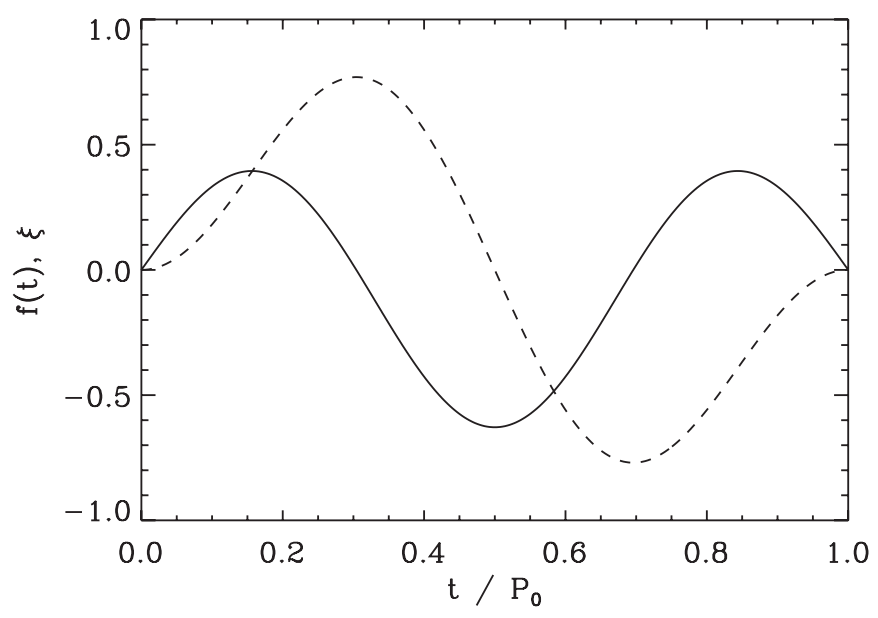

Figure 3. Time dependence of our driver, applied from $t=0$ to $t=P_{0}$. The dashed line represents the displacement $\xi$ of the flux tube axis (Equation (6)). The corresponding velocity dependence $f(t)$ is shown by the solid line (Equation (7)).

phase. The corresponding velocity dependence is calculated as the time derivative $f(t)=\frac{d \xi}{d t}$, i.e.,

$$
\begin{gathered}
\xi=\sin (\omega t) \sin \left(\frac{\omega}{2} t\right), 0 \leqslant t \leqslant P_{0} \\
f(t)=\omega \cos (\omega t) \sin \left(\frac{\omega}{2} t\right)+\frac{\omega}{2} \sin (\omega t) \cos \left(\frac{\omega}{2} t\right),
\end{gathered}
$$

where $\omega=2 \pi / P_{0}$ and the driver is applied from $t=0$ to $t=P_{0}$. The functions $f(t)$ and $\xi$ are shown in Figure 3 as solid and dashed lines, respectively. This driver generates a non-monochromatic propagating wavetrain with the dominant period of oscillation in a Fourier spectrum being $P \approx \frac{2}{3} P_{0}$.

The spatial dependence of the driver is based on a twodimensional dipole. We define a cylindrical core region with radius $r \leqslant b$, surrounded by a shell region $b<r \leqslant a$. In the core region, the velocity is constant $\mathbf{u}=\left(u_{0}, 0,0\right)$ and only in the $x$-direction. We choose $u_{0} / C_{A e}=0.003$ to be small to avoid nonlinear effects. In the surrounding environment $(r>a)$, we have the two-dimensional dipole form

$$
\mathbf{u}=u_{0} a^{2}\left(\frac{x^{2}-y^{2}}{\left(x^{2}+y^{2}\right)^{2}}, \frac{2 x y}{\left(x^{2}+y^{2}\right)^{2}}, 0\right) .
$$

The flow described so far corresponds to two-dimensional incompressible dipole flow around a circular tube that moves with velocity $\left(u_{0}, 0,0\right)$. In cylindrical coordinates, this would be described as the $m=1$ mode, in which $u_{r}$ is continuous and $u_{\phi}$ discontinuous at the tube boundary. To avoid numerical problems with velocity components that are discontinuous we change smoothly from the solution for the core to that for the environment in the shell region.

The simulations are performed using the MHD code LARE3D (Arber et al. 2001). The numerical domain is much larger in the $z$-direction than in $x$ or $y$, but the resolution is higher in the $x$-and $y$-directions in order to resolve the activity in the inhomogeneous layer for as long as possible, particularly when phase mixing takes place. Typical values used are $400 \times 400 \times 200$ grid points for a numerical domain of $4 \times 4 \times 20 \mathrm{Mm}(a=1 \mathrm{Mm})$.

The boundary conditions are periodic in the $x$ - and $y$-directions, and are placed sufficiently far away to not affect the results. Initially, the lower $z$ boundary is driven, but after

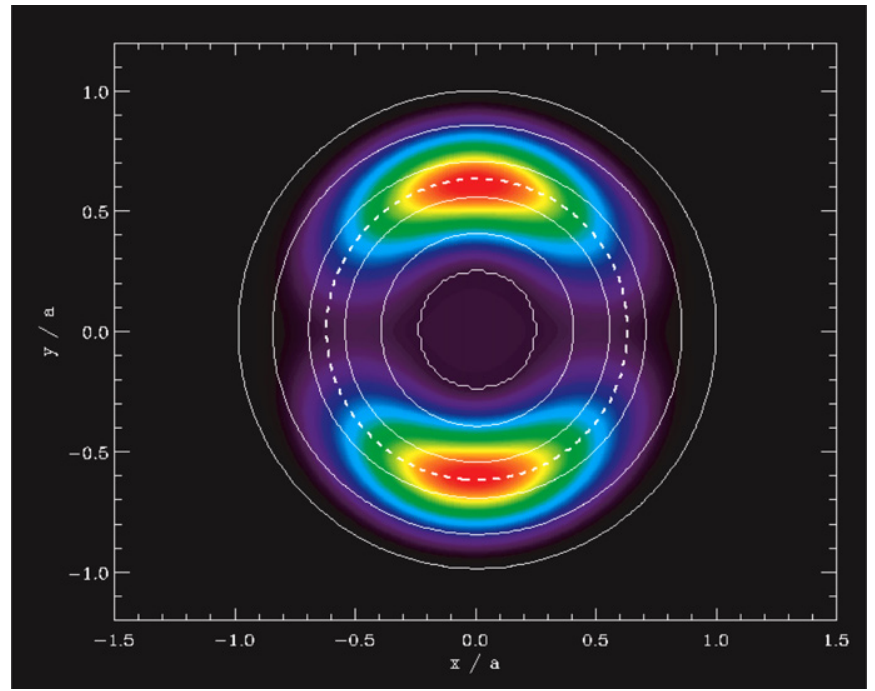

Figure 4. Wave energy integrated over direction of propagation $z$ at time $t=4 P_{0}$ for a wavepacket propagating through an undistorted cylinder. The solid lines are contours of density; the innermost and outermost contours show the edges of the core and shell regions, respectively. The dashed line represents the location where the mode coupling condition is satisfied.

(A color version of this figure is available in the online journal.)

our driving phase $\left(t \leqslant P_{0}\right)$ the driver is turned off and the $z$ boundaries also become periodic. This avoids the need for a large domain in the field-aligned direction $(z)$ by allowing the wavetrain to propagate out of the top of our domain and simply cycle through by re-entering at the lower boundary.

\section{RESULTS}

We wish to consider the case of a general nonuniform medium. In order to proceed in a tractable way we initially consider the case of a cylinder as in Pascoe et al. (2010) with distortions introduced only in the inhomogeneous (shell) region. We first consider a simple, symmetry decreasing azimuthal distortion, and then the case of a completely asymmetric cylinder with multiple distortions. Following these, we will move from the case of a single, cylindrical flux tube to a randomly generated inhomogeneous medium with multiple structures.

In our models, we consider a straight, uniform magnetic field in the $z$-direction. We use a zero plasma $\beta$ approximation.

\subsection{Non-axisymmetric Coronal Loops}

In our first case, the density profile describes a cylindrical tube aligned with the $z$-axis and defines three regions: the core region with an internal density $\rho_{0}$, the external or environment region with density $\rho_{e}$, and the inhomogeneous shell region in between, where the density varies linearly from $\rho_{0}$ to $\rho_{e}$ :

$$
\rho=\left\{\begin{aligned}
\rho_{0} & \text { if } r \leqslant b<a \\
\rho_{0}+\left(\rho_{e}-\rho_{0}\right)(r-b) / l & \text { if } b<r \leqslant a \\
\rho_{e} & \text { if } r>a,
\end{aligned}\right.
$$

where $r=\sqrt{x^{2}+y^{2}}$ and $l=a-b$. Here, we choose $a / b=4$ to provide a large shell region which is the main focus of our attention $(a=1 \mathrm{Mm})$. For $\rho_{0}>\rho_{e}$, the structure is a minimum in the Alfvén speed and so a waveguide for MHD waves (see, e.g., Edwin \& Roberts 1983; Roberts et al. 1984). We choose a density contrast $\rho_{0} / \rho_{e}=2$.

Figure 4 shows the wave energy (integrated over the entire $z$-range) at $t=4 P_{0}$ for the case of an undistorted cylinder. This 


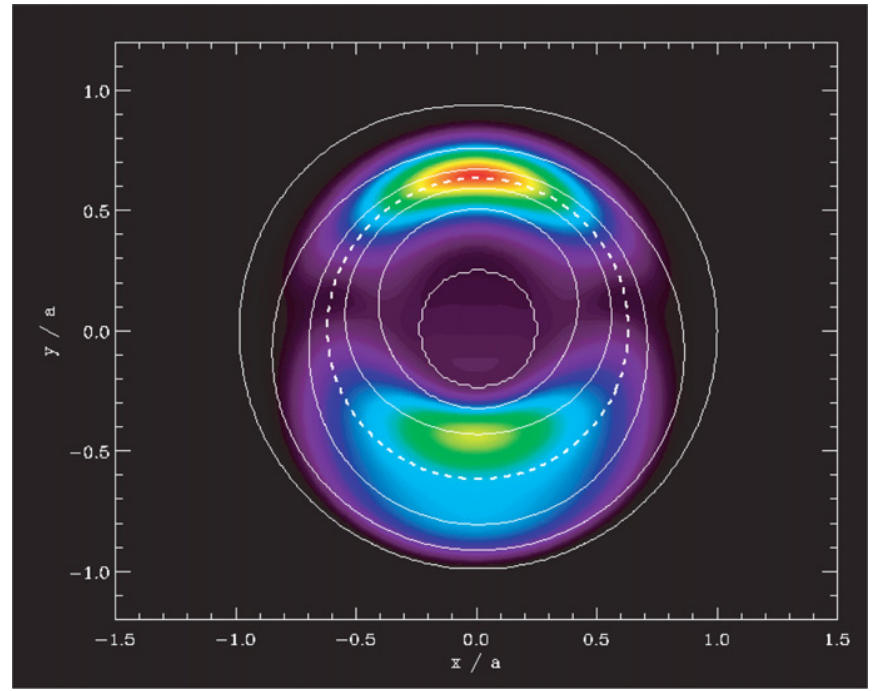

Figure 5. Wave energy integrated over $z$ at $t=4 P_{0}$ for a cylinder with an azimuthal distortion in the inhomogeneous region. Line contours as in Figure 4.

(A color version of this figure is available in the online journal.)

is the case studied in detail in Pascoe et al. (2010). The energy has been coupled in to Alfvén modes in the inhomogeneous region. The line contours represent density or Alfvén speed. Due to the symmetry of the cylindrical structure, the upper and lower mode coupling sites show the same behavior. The thick dashed line represents the contour for which the mode coupling condition is satisfied, i.e., $\omega=V_{A}(\mathbf{r}) k_{z}$, where $\omega$ is the dominant (angular) frequency of the kink wavepacket, $V_{A}(\mathbf{r})$ is the local Alfvén speed, and $k_{z}$ is the local longitudinal wavenumber. The wave energy is centered on this contour and the two maxima represent the two antinodes of the azimuthally polarized Alfvén mode. Wave energy is located all along this contour, except at the two nodes, which are located at $y=0$, which correspond to nodes in the magnetic pressure gradient of the kink mode (Russell \& Wright 2010).

The first type of deformation we consider is an azimuthal perturbation to the density of the form $\sin \theta$. Figure 5 shows the wave energy integrated over $z$ for this case. The distortion is added such that the density in the inhomogeneous layer never rises above the core density $\rho_{0}$ or falls below the external density $\rho_{e}$. This distortion has the effect of increasing the gradient in the inhomogeneous layer at the upper $(y>0)$ mode coupling site, and decreasing the gradient at the lower $(y<0)$ mode coupling site. The wave energy is centered on the dashed contour but is more localized for the upper mode coupling site than for the lower site due to the larger gradient there.

Finally, Figure 6 shows the wave energy integrated over $z$ for a cylinder with multiple distortions. The distortions are generated by adding Gaussian structures of varying sizes and locations to the density profile in the inhomogeneous layer. The distortions are added such that the total mass is conserved with respect to the undistorted case, and the density in the inhomogeneous layer never rises above the core density $\rho_{0}$ or falls below the external density $\rho_{e}$. Once again, the locations of the wave energy maxima can be seen to be centered on the dashed contour where the mode coupling condition is satisfied, and the wave energy is more concentrated in regions of higher gradient.

Figure 7 shows the (normalized) wave energy in the core region integrated over the entire $z$ domain. This has the advantage that the only wave perturbations in the uniform core region

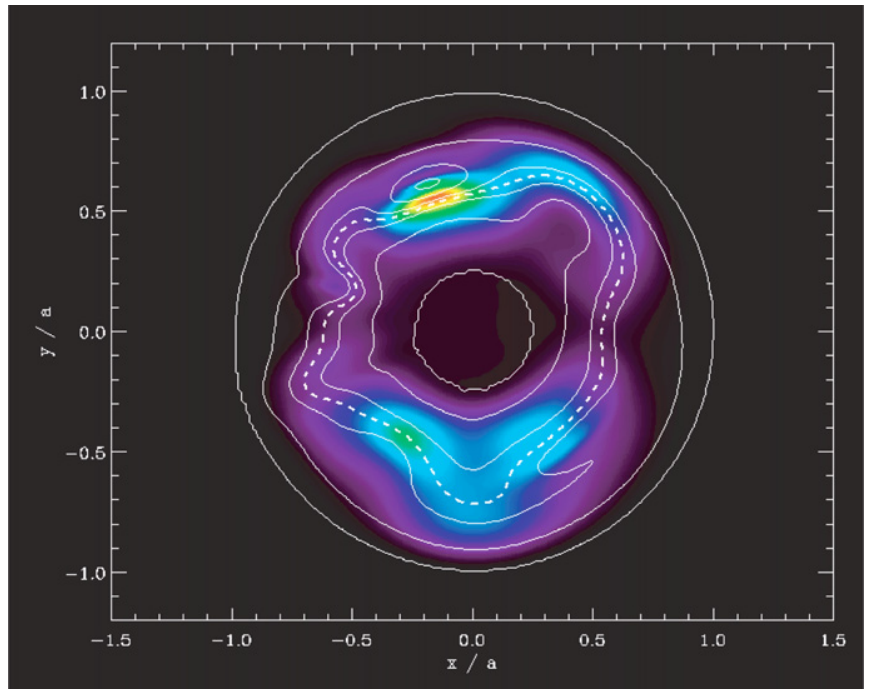

Figure 6. Wave energy integrated over $z$ at $t=4 P_{0}$ for a cylinder with multiple distortions to the density profile in the inhomogeneous region. Line contours as in Figure 4.

(A color version of this figure is available in the online journal.)

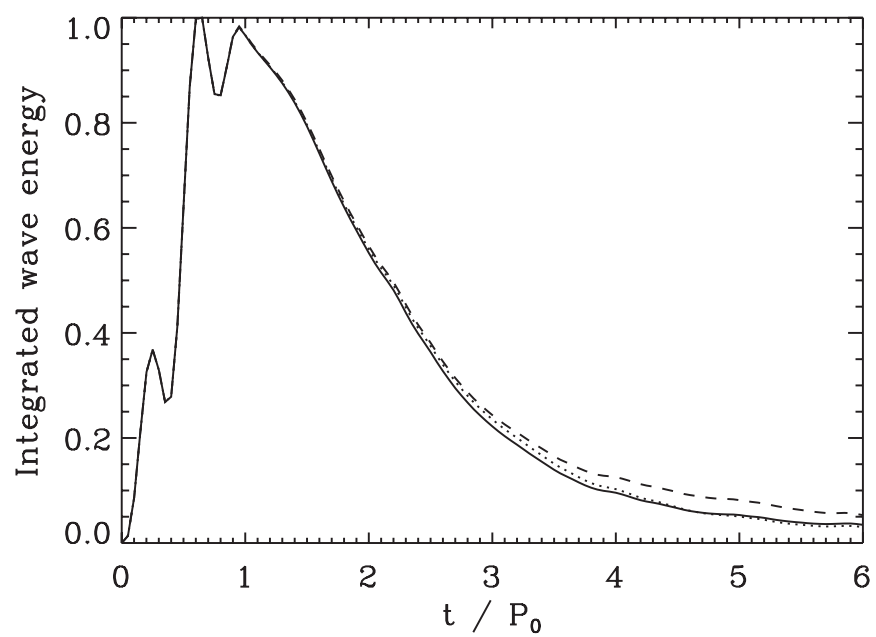

Figure 7. Wave energy integrated over the core region for the three cylindrical structures in Figures 4-6. The solid line shows the case of the undistorted cylinder. The dashed and dotted lines represent the cylinders with azimuthal and random distortions, respectively.

will be kink waves, and allow a direct way of identifying them and their decay (through mode coupling to Alfvén waves). The solid line shows the case of the undistorted cylinder. The dashed and dotted lines represent the cylinders with azimuthal and random distortions, respectively. The wave energy increases during the driving phase to a maximum value at $t=P_{0}=10 \mathrm{~s}$. Once the driver stops, the energy in the core region decreases exponentially as energy is transferred from the kink mode in the core region, to the Alfvén mode in the inhomogeneous shell region. The decay rate of the kink mode shows little sensitivity to the details of density structuring.

\subsection{Randomly Structured Medium}

Next, we consider a randomly structured corona where the driver is no longer prescribed to be aligned with a particular structure. The density profile is generated as the sum of 10 Gaussian structures, each with a random position, height, and width. The density profile is shown in Figure 8. Each individual 

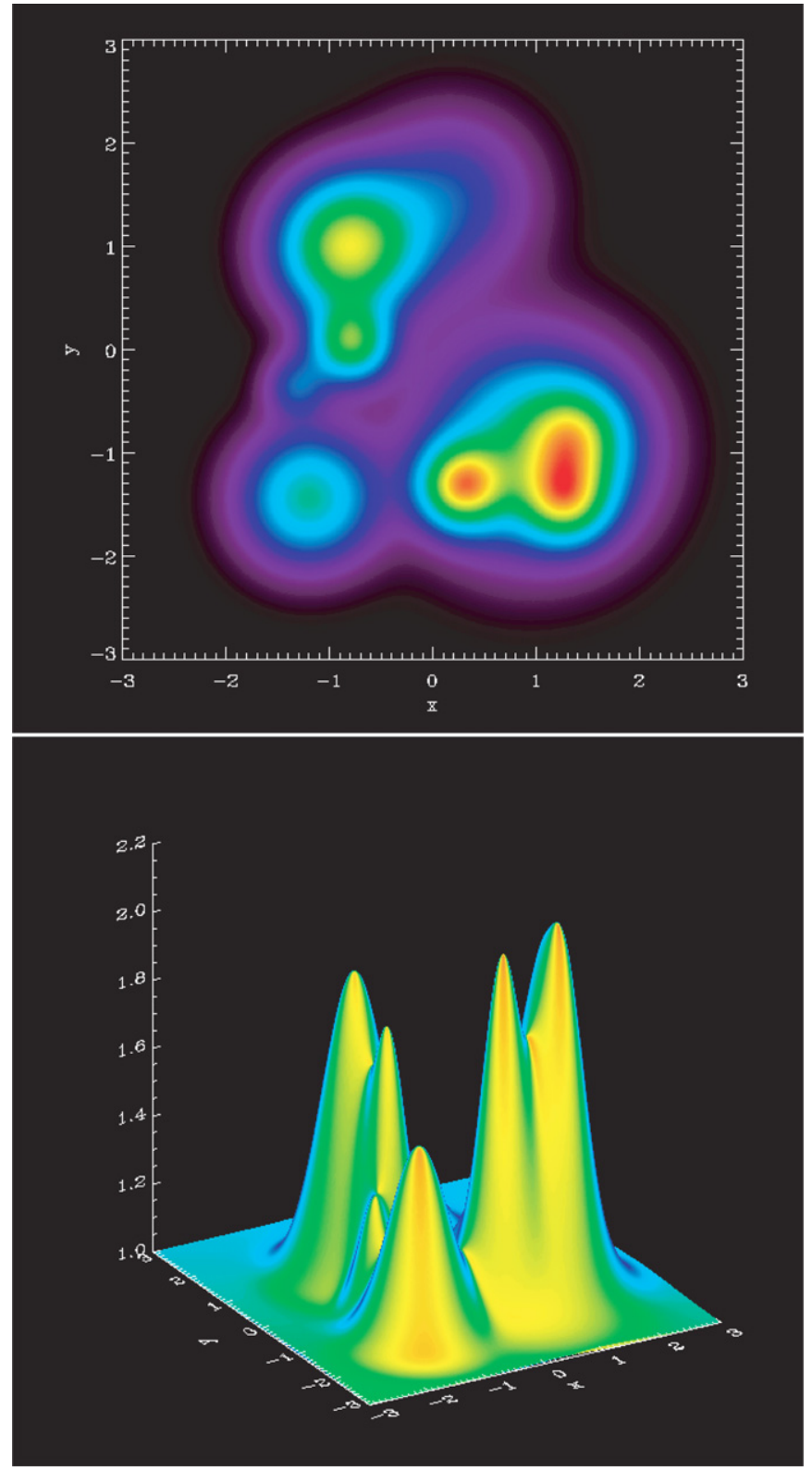

Figure 8. Density profile for randomly structured medium composed of Gaussian density enhancements.

(A color version of this figure is available in the online journal.)

Gaussian structure can be considered to be the equivalent of the cylinder in Equation (9) with $l=a$ (i.e., $b=0$ ) and having a nonlinear gradient in $r$. The driver remains the same as in the simulations considered in Section 4.1 (with $a / b=2$ ); however, it is now no longer associated with any particular structure, and in the case shown in Figure 8 it is actually centered on a region of low density.

Figure 9 shows the wave energy density integrated over $z$ at time $t=P_{0}=10 \mathrm{~s}$, i.e., just after the driver stops, and at $4 P_{0}$. The lines show the density profile contours. At early times, the wave energy is concentrated in the driven wavepacket, approximately in a cylinder of radius $a=1 \mathrm{Mm}$ centered on $x=y=0$. At later times, the wave energy shows four maxima (labeled 1-4). The dashed line represents the location where the local mode coupling condition is satisfied, i.e., the kink and Alfvén phase speeds are equal, where the local density
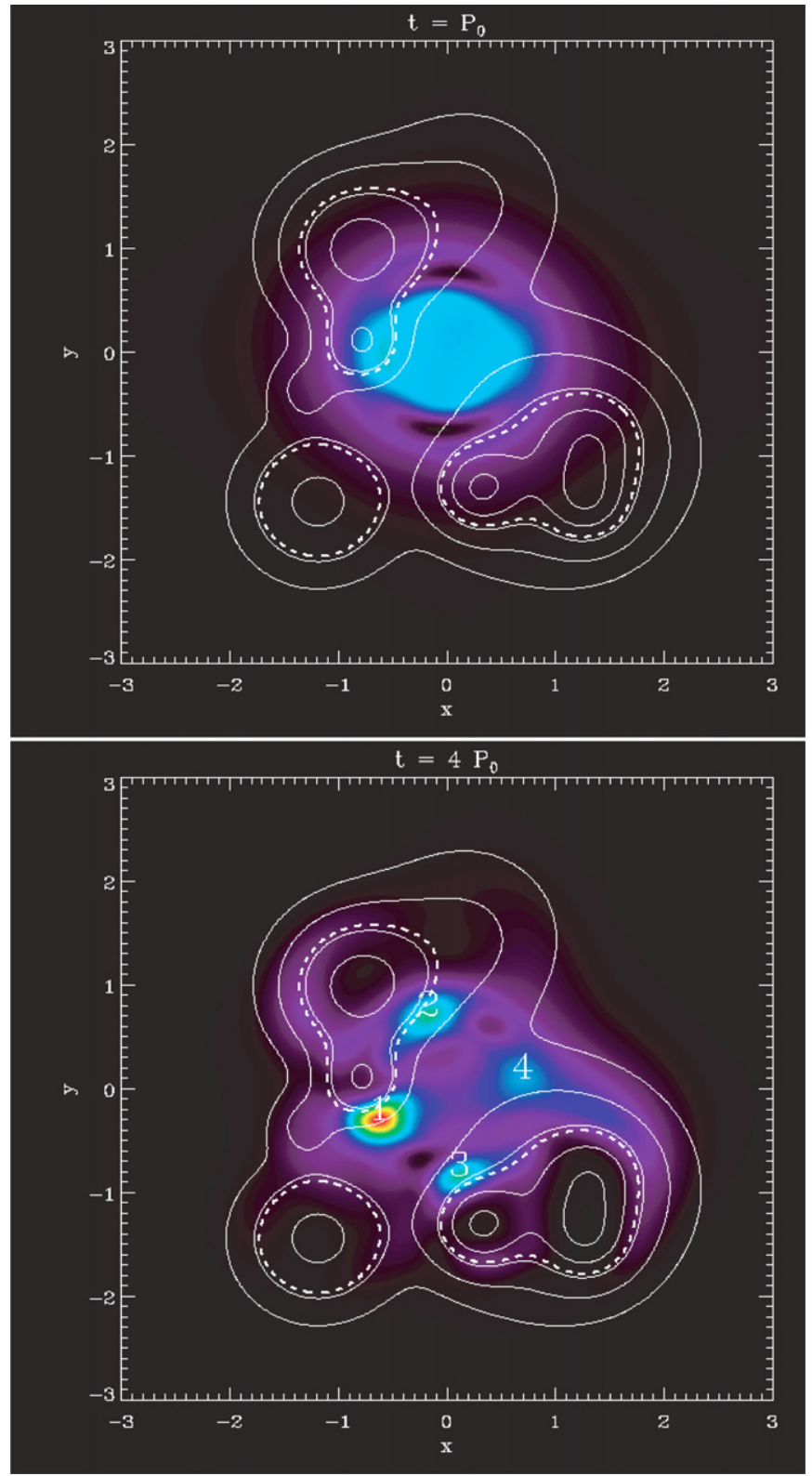

Figure 9. Wave energy (integrated over $z$ ) at $t=P_{0}$ (top) and $t=4 P_{0}$ (bottom) The wave energy is initially dominated by the dipole flow of the driver, whereas at later times four maxima are seen to emerge. The dashed line represents the location where the local mode coupling condition is satisfied.

(A color version of this figure is available in the online journal.)

maximum is used to calculate the kink phase speed

$$
C_{k}=\left(\frac{\rho_{0} C_{A 0}^{2}+\rho_{e} C_{A e}^{2}}{\rho_{0}+\rho_{e}}\right)^{1 / 2}
$$

where $C_{A 0}=\left(B_{0}^{2} / \mu \rho_{0}\right)^{1 / 2}$ and $C_{A e}=\left(B_{e}^{2} / \mu \rho_{e}\right)^{1 / 2}$. This simple estimate of the coupling location is merely intended as a rough guide as the medium can, at best, only locally be described as a distorted cylinder. Despite this obvious limitation, there is a reasonable correspondence with the locations of wave energy maxima.

Figure 10 shows a cut in $v_{x}$ at $y=0$ and at times $t=P_{0}$ (left), $t=4 P_{0}$ (middle), and $t=8 P_{0}$ (right) for a wavetrain propagating through the inhomogeneous medium in Figure 8. The contours in each panel are plotted with the same scale. At 


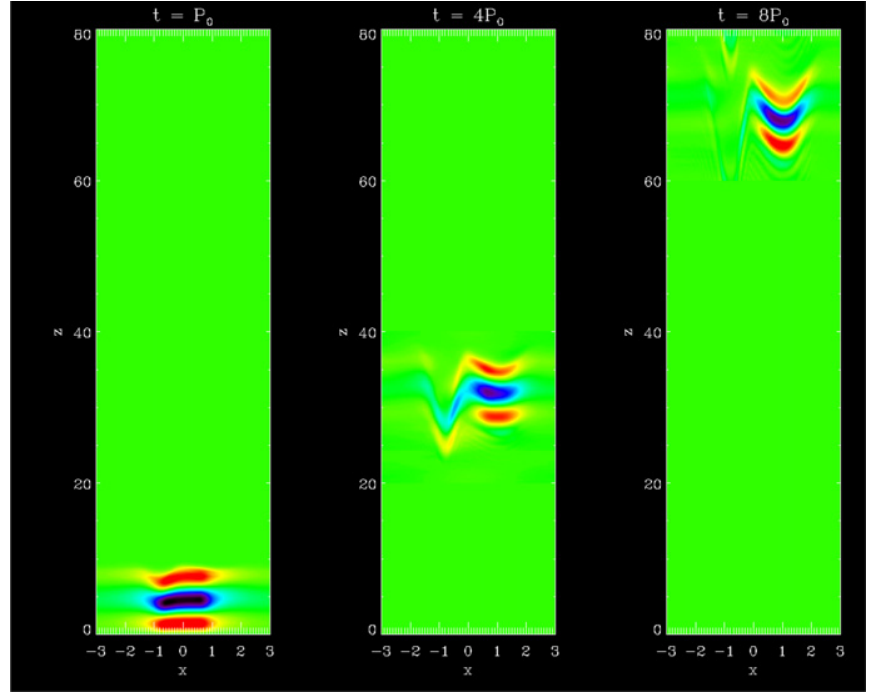

Figure 10. $v_{x}(x, y=0, z)$ at times $t=P_{0}$ (left), $t=4 P_{0}$ (middle), and $t=8 P_{0}$ (right) for a wavetrain propagating through the inhomogeneous medium in Figure 8.

(A color version of this figure is available in the online journal.)

early times, the wavetrain has a well-defined structure consisting of three anti-nodes, reflecting the driver applied at the lower boundary (see Figure 3 ). The numerical domain has a length of $20 \mathrm{Mm}$ in the $z$-direction, with periodic boundary conditions at the upper and lower boundaries after the driving phase. Here, we have shifted the cuts in $v_{x}$ vertically in order to highlight the propagating nature of the wavetrain, i.e., in the middle panel the wavetrain has propagated through the periodic $z$ boundaries once, and in the right panel it has propagated through three times.

The left part of the wavetrain propagates through a region where the mode coupling condition is satisfied (near maximum 1 in Figure 9) and so shows significant attenuation. The right part of the wavetrain propagates near maximum 4 in Figure 9, where the medium is only weakly nonuniform and so the wavetrain shows little attenuation but does become distorted at later times due to the gently varying Alfvén speed.

Figure 11 shows the integrated wave energy at the four maxima in Figure 9. In all cases, the wave energy increases from $t=0$ to $t=P_{0}$ during the driving phase. Subsequently, three of the cases (maxima 1-3 in Figure 9) show an increase in wave energy with time until reaching saturation at approximately $t=6 P_{0}$. In the case of the fourth maximum (maximum 4 in Figure 9), the wave energy instead remains approximately constant at the level of the driven energy.

The growth of energy at the three maxima resembles the concentration of energy in the inhomogeneous layer in the case of a cylindrical density structure considered in Section 4.1 and Pascoe et al. (2010). This suggests these are sites of mode conversion, as expected from the qualitative agreement with the estimated location based on phase speeds (Figure 9). This result is analogous to the study of Terradas et al. (2008). These authors investigate standing modes in an inhomogeneous flux tube and found that the coupling between fast and Alfvén waves is not affected by the complicated geometry. They are also located close to the region where the driver acts. It is likely that there are other locations in the profile where the condition for mode conversion is satisfied, but where the driven wave energy is too low to produce significant concentrations, or nodes in the

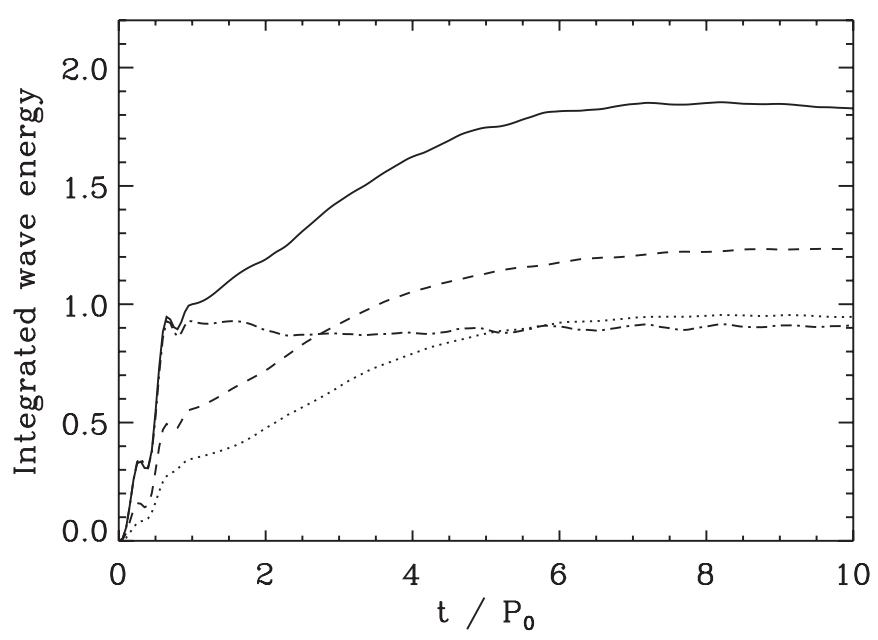

Figure 11. Wave energy (integrated over $z$ ) for the four maxima seen in the lower panel of Figure 9. The solid, dashed, dotted, and dash-dotted lines correspond to maxima $1-4$, respectively.

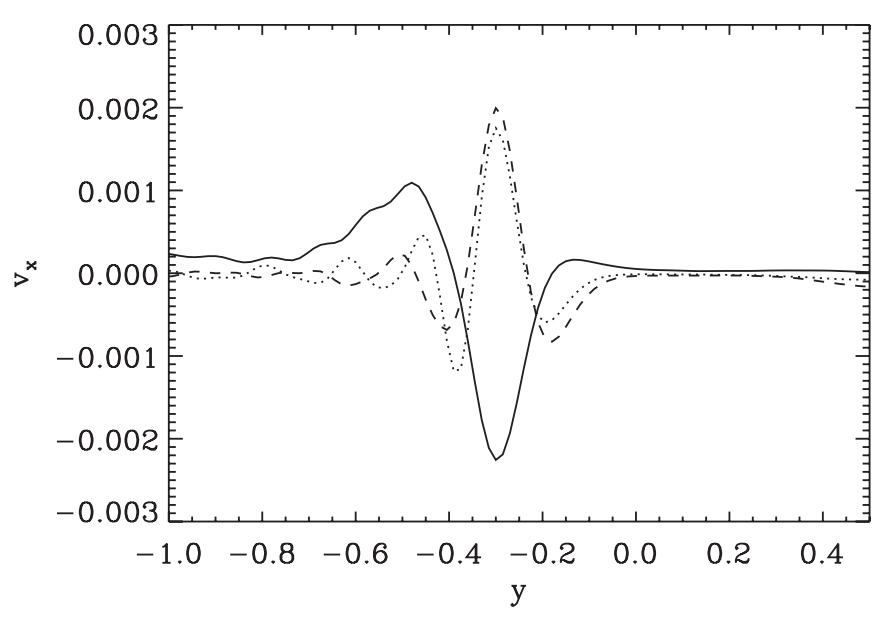

Figure 12. Phase mixing: the solid, dashed, and dotted lines show $v_{x}$ at $t=6 P_{0}$, $8 P_{0}$, and $10 P_{0}$, respectively.

Alfvén wave energy may occur, as in the intersections of the mode coupling contour and the $x$-axis in Figure 4. An example of such a location is the density concentration in the bottom left of Figure 9 where no wave energy accumulates.

Since the Alfvén modes are in an inhomogeneous layer, they are subject to phase mixing. This can be seen in Figure 12 which shows $v_{x}$ as a function of the transverse direction $y$ through maximum 1 (at $x=-0.6$ ) in Figure 9. The solid, dashed, and dotted lines show $v_{x}$ at time $t=6 P_{0}, 8 P_{0}$, and $10 P_{0}$, respectively. At later times, the phase mixing length scale is seen to decrease, corresponding to the development of larger gradients.

In order to support our interpretation of the wave energy maxima in Figure 9 as Alfvén modes, including the case of the maximum that shows no growth, we look at the transverse components of velocity and magnetic field at these four locations as a function of longitudinal direction $z$ at $t=4 P_{0}$. We use the normalized Alfvén wave relations,

$$
b_{x}+\rho^{1 / 2} v_{x}=0, b_{y}+\rho^{1 / 2} v_{y}=0, b_{z}=0,
$$

where $\mathbf{b}=\mathbf{B}-\mathbf{B}_{t=0}$. Figure 13 shows $\rho^{1 / 2} v_{x, y},-b_{x, y}$ and $b_{z}$ as solid, dashed, and dotted lines, respectively. The overlap of the solid and dashed lines, and the negligible values of the dotted 

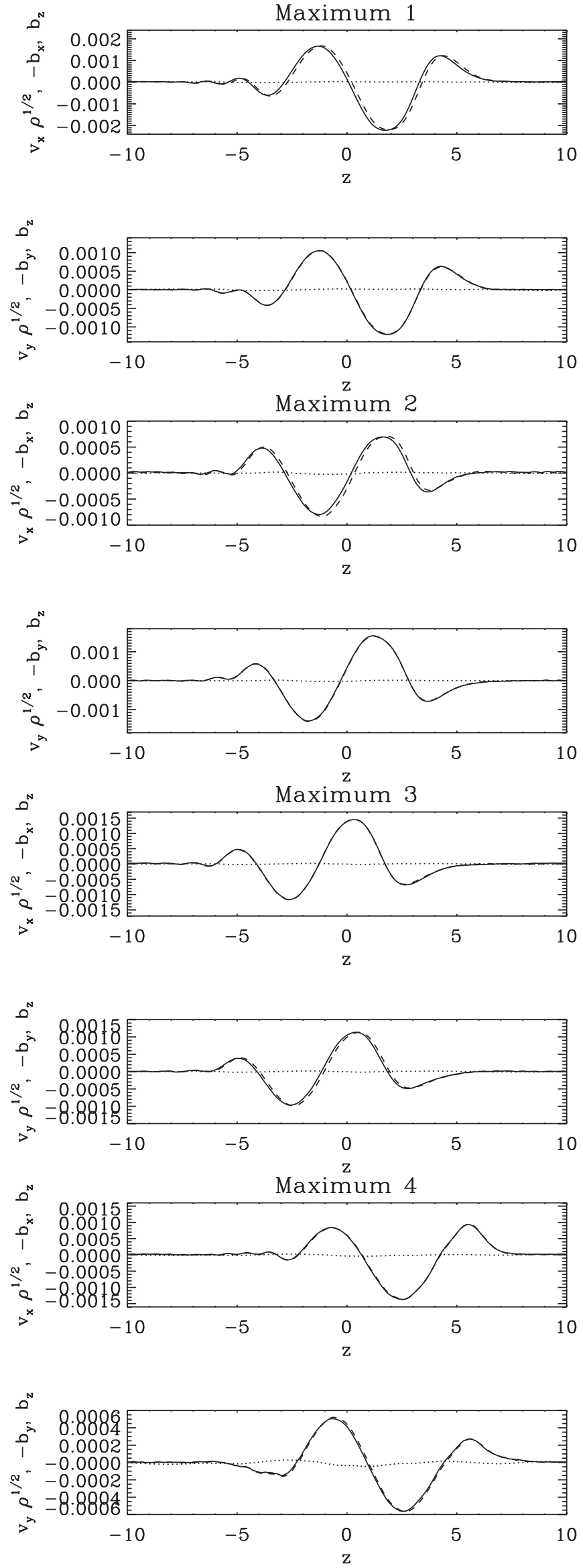

Figure 13. Alfvén mode diagnostics. Wave fields plotted as a function of $z$ at $t=4 P_{0}$ for the four maxima seen in the lower panel of Figure 9.

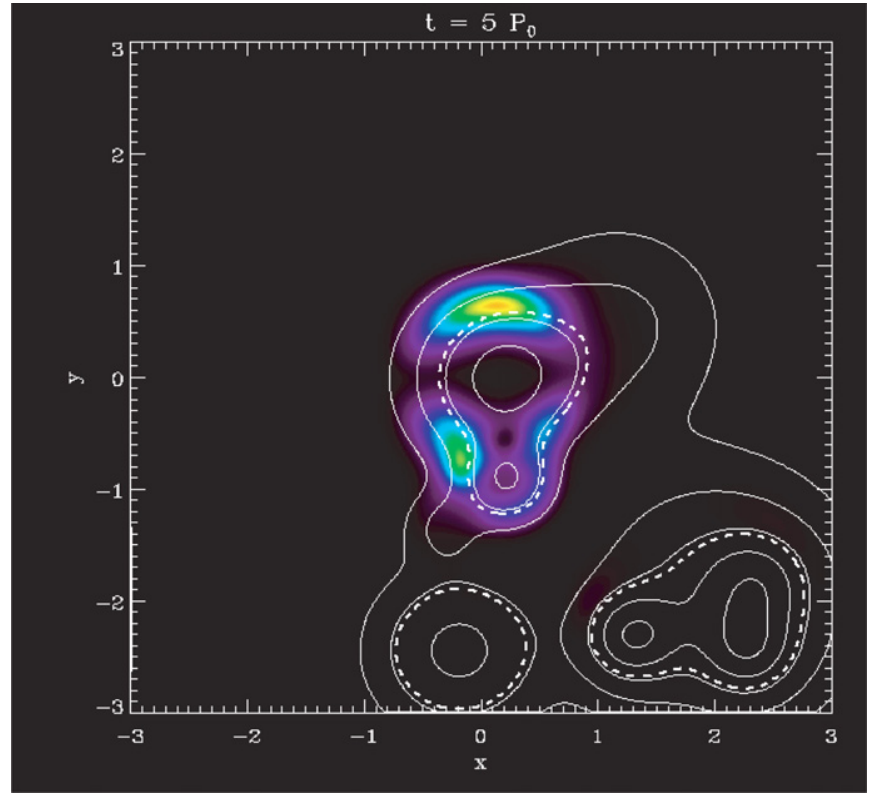

Figure 14. Wave energy (integrated over $z$ ) at $t=5 P_{0}$ for the shifted density profile. Line contours as in Figure 9.

(A color version of this figure is available in the online journal.)

lines represent the solution of Equation (11). This suggests the wave energy maximum that does not show any growth (maximum 4) is an Alfvén mode excited by the driver directly (e.g., Tirry \& Berghmans 1997; De Groof et al. 2002) rather than by mode coupling from the kink mode. This is also consistent with the maximum being far from the locations where the local mode coupling condition is satisfied (Figure 9). Throughout the simulation this maximum propagates without growth or decay following the driving phase, as Figure 11 demonstrates clearly. It is interesting to see that even Alfvén waves that do not have sufficient symmetry to be decoupled are able to survive without decay for the duration of our simulation. A review of Alfvén waves excited both directly and indirectly by footpoint motions can be found in Goossens \& De Groof (2001).

In the model described so far, the driver was aligned with a local minimum in the density. Figure 14 shows the wave energy (integrated over $z$ ) at $t=5 P_{0}$ for the same randomly generated density profile but shifted such that the driver is now aligned with one of the local density maxima. It is clear that the wave energy remains near this local structure with the results strongly resembling the case of the distorted cylinder discussed in Section 4.1.

\section{DISCUSSION}

We have considered the propagation of a wavetrain through an inhomogeneous low $\beta$ plasma. In Section 4.1, we moved from a case of an ideal cylindrical structure to an increasingly distorted cylinder. In Section 4.2, we applied the same ideas to an arbitrary inhomogeneous two-dimensional density profile.

When the density structure could be regarded as a flux tube (albeit distorted) with a uniform core region, we could clearly see how the kink oscillations present in the core decayed through mode conversion to Alfvén waves. This gave an estimate of the damping time of the kink wavepacket, and hence the timescale on which energy is transferred to the Alfvén waves. The three tubes considered all showed similar timescales. When we considered the randomly structured medium, it was less easy 
to get an unambiguous measure of the fast (kink) mode energy and monitor its decay through wave coupling. However, it is easy to monitor the Alfvén wave energy at the sites of strong coupling, and we see this shows a rise in energy (after the driving phase), presumably as fast-mode energy is coupled in to (i.e., tranferred to) the Alfvén waves. Thus, we have direct evidence of wave coupling, this time from wave growth rather than wave decay. The timescales for wave coupling in Figures 7 and 11 are similar, despite vastly different density structures (for comparison, the total mass in the randomly structured medium is $\approx 11 \%$ higher than for the distorted cylinders).

The Alfvén wave energy enhancement in Figure 9 labeled as maximum 4 shows no growth following the driving phase (Figure 11). This is probably because it is located close to a maximum in the Alfvén speed. Consequently, the medium is, locally, almost uniform and hence decoupled wave modes are the appropriate solutions. Hence, the Alfvén wave energy deposited here during the driving phase simply remains as a decoupled Alfvén wave.

Further evidence of the role of fast/kink-like waves can be seen in Figure 9. The energy distribution in the two panels is different. In particular, it has redistributed itself across field lines. The Alfvén wave cannot transport energy perpendicular to the magnetic field, so this must be accomplished by the presence of fast-mode perturbations (such as kink oscillations in the axisymmetric models).

In all cases considered, modest changes in density (i.e., local density enhancements comparable in magnitude to the background) were capable of efficiently converting energy from the driving footpoint motion to localized Alfvén modes. We have demonstrated that mode conversion efficiently couples kink-like perturbations to Alfvén modes in an arbitrary inhomogeneous medium. In fact, comparing Figures 9 and 14 shows that the mode coupling is equally efficient whether the driver is centered on a local density maximum or not. This suggests that any transverse footpoint motion at the base of the corona will effectively deposit energy in Alfvén modes in the corona. Since these Alfvén modes will then phase-mix, the footpoint motion energy eventually becomes localized to small scales and so may contribute to coronal heating, which we will investigate in a future paper.

D.J.P. acknowledges financial support from STFC. I.D.M. acknowledges support of a Royal Society University Research Fellowship. The computational work for this paper was carried out on the joint STFC and SFC (SRIF) funded cluster at the University of St Andrews (Scotland, UK).

\section{REFERENCES}

Allan, W., White, S. P., \& Poulter, E. M. 1986, Planet. Space Sci., 34, 371

Allan, W., \& Wright, A. N. 1998, J. Geophys. Res., 103, 2359

Allan, W., \& Wright, A. N. 2000, J. Geophys. Res., 105, 317
Andries, J., van Doorsselaere, T., Roberts, B., Verth, G., Verwichte, E., \& Erdélyi, R. 2009, Space Sci. Rev., 149, 3

Arber, T. D., Longbottom, A. W., Gerrard, C. L., \& Milne, A. M. 2001, J. Comput. Phys., 171, 151

Arregui, I., Andries, J., Van Doorsselaere, T., Goossens, M., \& Poedts, S. 2007a, A\&A, 463, 333

Arregui, I., Terradas, J., Oliver, R., \& Ballester, J. L. 2007b, A\&A, 466, 1145

Berghmans, D., \& Tirry, W. J. 1997, A\&A, 325, 318

Cirtain, J. W., et al. 2007, Science, 318, 1580

Davila, J. M. 1987, ApJ, 317, 514

De Groof, A., \& Goossens, M. 2000, A\&A, 356, 724

De Groof, A., \& Goossens, M. 2002, A\&A, 386, 691

De Groof, A., Paes, K., \& Goossens, M. 2002, A\&A, 386, 681

De Moortel, I. 2005, Phil. Trans. R. Soc. A, 363, 2743

De Pontieu, B., et al. 2007, Science, 318, 1574

Edwin, P. M., \& Roberts, B. 1983, Sol. Phys., 88, 179

Erdélyi, R., \& Fedun, V. 2007, Science, 318, 1572

Goossens, M. 2008, in IAU Symp. 247, Waves \& Oscillations in the Solar Atmosphere: Heating and Magneto-Seismology, ed. R. Erdélyi \& C. A. Mendoza-Briceño (Dordrecht: Kluwer), 228

Goossens, M., Andries, J., \& Arregui, I. 2006, Phil. Trans. R. Soc. A, 364, 433

Goossens, M., Andries, J., \& Aschwanden, M. J. 2002, A\&A, 394, L39

Goossens, M., \& De Groof, A. 2001, Phys. Plasmas, 8, 2371

Goossens, M., Hollweg, J. V., \& Sakurai, T. 1992, Sol. Phys., 138, 233

Goossens, M., Terradas, J., Andries, J., Arregui, I., \& Ballester, J. L. 2009, A\&A, 503,213

Hollweg, J. V. 1987, ApJ, 312, 880

Hollweg, J. V. 1990, Comput. Phys. Rep., 12, 205

Hollweg, J. V., \& Yang, G. 1988, J. Geophys. Res., 93, 5423

Hood, A. W., Brooks, S. J., \& Wright, A. N. 2005, Proc. R. Soc. A, 461, 237

Ionson, J. A. 1978, ApJ, 226, 650

Keppens, R., Bogdan, T. J., \& Goossens, M. 1994, ApJ, 436, 372

Nakariakov, V. M., \& Verwichte, E. 2005, Living Reviews in Solar Physics 2, http://www.livingreviews.org/lrsp-2005-3 (cited 2007 August)

Ofman, L., Davila, J. M., \& Steinolfson, R. S. 1994, ApJ, 421, 360

Ofman, L., Davila, J. M., \& Steinolfson, R. S. 1995, ApJ, 444, 471

Pascoe, D. J., Wright, A. N., \& De Moortel, I. 2010, ApJ, 711, 990

Poedts, S. 2002, in IAU Colloq. 188, SOLMAG 2002, Proc. Magnetic Coupling of the Solar Atmosphere Euroconference, ed. H. Sawaya-Lacoste (ESA SP505; Noordwijk: ESA), 273

Roberts, B. 2008, in IAU Symp. 247, Waves \& Oscillations in the Solar Atmosphere: Heating and Magneto-Seismology, ed. R. Erdélyi \& C. A. Mendoza-Briceño (Dordrecht: Kluwer), 3

Roberts, B., Edwin, P. M., \& Benz, A. O. 1984, ApJ, 279, 857

Ruderman, M. S., \& Roberts, B. 2002, ApJ, 577, 475

Russell, A. J. B., \& Wright, A. N. 2010, A\&A, 511, A17

Steinolfson, R. S., \& Davila, J. M. 1993, ApJ, 415, 354

Terradas, J., Arregui, I., Oliver, R., \& Ballester, J. L. 2008, ApJ, 679, 1611

Terradas, J., Goossens, M., \& Verth, G. 2010, A\&A, 524, A23

Terradas, J., Oliver, R., \& Ballester, J. L. 2006, ApJ, 642, 533

Tirry, W. J., \& Berghmans, D. 1997, A\&A, 325, 329

Tirry, W. J., Berghmans, D., \& Goossens, M. 1997, A\&A, 322, 329

Tomczyk, S., \& McIntosh, S. W. 2009, ApJ, 697, 1384

Tomczyk, S., McIntosh, S. W., Keil, S. L., Judge, P. G., Schad, T., Seeley, D. H., \& Edmondson, J. 2007, Science, 317, 1192

Van Doorsselaere, T., Nakariakov, V. M., \& Verwichte, E. 2008, ApJ, 676, L73

Verth, G., Terradas, J., \& Goossens, M. 2010, ApJ, 718, L102

Walsh, R. W., \& Ireland, J. 2003, A\&AR, 12, 1

Wright, A. N., \& Allan, W. 2008, J. Geophys. Res., 113, A02206

Wright, A. N., Allan, W., Elphinstone, R. D., \& Cogger, L. L. 1999, J. Geophys. Res, 104, 10159

Wright, A. N., \& Rickard, G. J. 1995, ApJ, 444, 458 